\title{
Testing the Universality of Free Fall with the Triple System J0337+1715
}

\author{
A. M. Archibald ${ }^{1}$, N. V. Gusinskaia ${ }^{1}$ J. W. T. Hessels ${ }^{1,2}$, \\ D. R. Lorimer ${ }^{3,4,5}$, R. S. Lynch ${ }^{4,5}$, S. M. Ransom ${ }^{6}$ and I. H. Stairs ${ }^{7}$ \\ ${ }^{1}$ Anton Pannekoek Institute for Astronomy, University of Amsterdam, Science Park 904, 1098 \\ XH Amsterdam, The Netherlands \\ email: A.Archibald@uva.nl \\ ${ }^{2}$ ASTRON, the Netherlands Institute for Radio Astronomy, Postbus 2, 7990 AA, Dwingeloo, \\ The Netherlands \\ ${ }^{3}$ Department of Physics and Astronomy, West Virginia University, PO Box 6315, Morgantown, \\ WV 26506, USA \\ ${ }^{4}$ Center for Gravitational Waves and Cosmology, West Virginia University, Chestnut Ridge \\ Research Building, Morgantown, WV 26505, USA \\ ${ }^{5}$ Green Bank Observatory, Green Bank, WV 24944, USA \\ ${ }^{6}$ National Radio Astronomy Observatory, Charlottesville, VA 22903, USA \\ ${ }^{7}$ Department of Physics and Astronomy, University of British Columbia, Vancouver, BC V6T \\ 1Z1, Canada
}

\begin{abstract}
The millisecond pulsar PSR J0337+1715 is in a mildly relativistic hierarchical triple system with two white dwarfs. This offers the possibility of testing the universality of free fall: does the neutron star fall with the same acceleration as the inner white dwarf in the gravity of the outer white dwarf? We have carried out an intensive pulsar timing campaign, yielding some 27000 pulse time-of-arrival (TOA) measurements with a median uncertainty of $1.2 \mu$ s. Here we describe our analysis procedure and timing model.
\end{abstract}

Keywords. gravitation, relativity, methods: data analysis, methods: numerical, methods: statistical, (stars:) pulsars: individual (PSR J0337+1715)

The Strong Equivalence Principle (SEP), the idea that all experiments including gravitational ones give the same result in any inertial frame, is a key ingredient in Einstein's theory of general relativity, and essentially all alternative theories violate it at some level. The SEP requires local position invariance, local Lorentz invariance, and the universality of free fall, that is, the idea that all objects fall the same way in a gravitational field. Laboratory experiments have tested the composition independence of free fall to very high accuracy, but it is infeasible to construct objects with substantial amounts of gravitational binding energy in the laboratory. Lunar laser ranging is able to test whether the Earth and Moon fall the same way in the Sun's gravity, but the Earth's fractional gravitational binding energy is at the $10^{-9}$ level, limiting how well the experiments are able to constrain theory. By contrast, the pulsar PSR J0337+1715 is expected to have gravitational binding energy $10-15 \%$ of its mass. We therefore study the system to carry out a sensitive strong-field test of the universality of free fall.

Our basic technique is that of pulsar timing: from radio observations we construct pulse time-of-arrival (TOA) data, we build a timing model that describes all relevant physics, 
and we use a least-squares fitting operation to compute the best-fit model parameters. Finally we use a Fourier-domain method to estimate systematics remaining in the data.

\section{Data and initial processing}

Our raw data consists of observations of the system with the Westerbork Synthesis Radio Telescope (WSRT), the Robert C. Byrd telescope at Green Bank (GBT), and the Arecibo Observatory telescope (AO). The observations were recorded with the pulsar backends PuMaII, GUPPI, and PUPPI respectively. These backends carry out coherent dedispersion and online folding based on an approximate long-term ephemeris, producing a folded profile every 10 seconds for each channel (approximately $1.6 \mathrm{MHz}$ ). Altogether we have approximately 800 observations, corresponding to roughly 1200 hours of time on sky. The majority of our observations are in the $1400 \mathrm{MHz}$ band, though a smaller fraction are recorded at $430 \mathrm{MHz}$ or $350 \mathrm{MHz}$.

Our data reduction pipeline approximately follows that used by the NANOGrav pulsar timing array project (The NANOGrav Collaboration et al. 2015). We augmented the automatic radio-frequency interference excision with a certain amount of manual flagging. Because it is infeasible to use the full ephemeris for live observations, we realign all observations according to an up-to-date timing model. To ensure adequate frequency resolution to handle scintillation while also obtaining adequate signal-to-noise, we average each observation to give a pulse profile each 20 minutes for each $20 \mathrm{MHz}$. These folded profiles consist of nominally calibrated Stokes IQUV parameters, but we were unable to calibrate our Arecibo observations well enough to work with Stokes I only (see Figure 1). We therefore selected the highest signal-to-noise GBT observation and constructed a single smoothed template profile (also containing full polarization information). We used a Mueller-matrix fitting procedure like that of van Straten (2006) to obtain the phase alignment and polarization transformation that best match this template to each observed profile. This phase alignment yields a TOA at the center of each subintegration. We use a long-term ephemeris to assign the correct pulse number to each of these TOAs.

\section{Timing model}

Neither a closed formula nor a series approximation of adequate accuracy is known to model the motion of this three-body system. We therefore build a timing model by direct integration of the equations of motion. We previously (Ransom et al. 2014) used simple Newtonian equations of motion, but we now use a parameterized post-Newtonian Lagrangian (Nordtvedt 1985), from which we extract equations of motion using the symbolic algebra package sympy. We use a two-non-interacting-Keplerian model to parameterize the initial conditions of the system. For each initial configuration, we use a Bulirsch-Stoer differential equation solver (from the software library odeint inside boost) to compute the orbit of all three bodies. At each integrator step, we compute the time an emitted pulse would reach the Solar System barycenter, and when this time passes the (barycentered) time of a TOA, we use a root-finding algorithm to determine the emission time of the pulse in pulsar proper time.

Given the pulse emission time for each TOA, a linear least-squares problem remains to determine the best-fit pulsar spin period and period derivative. Also included in this part of the fit are many other parameters that are approximately or exactly linear: instrument-dependent delays, pulsar position, proper motion, and parallax errors, as well as dispersion measure variations. We are also able to analytically marginalize over these linear parameters in a Bayesian fit. 

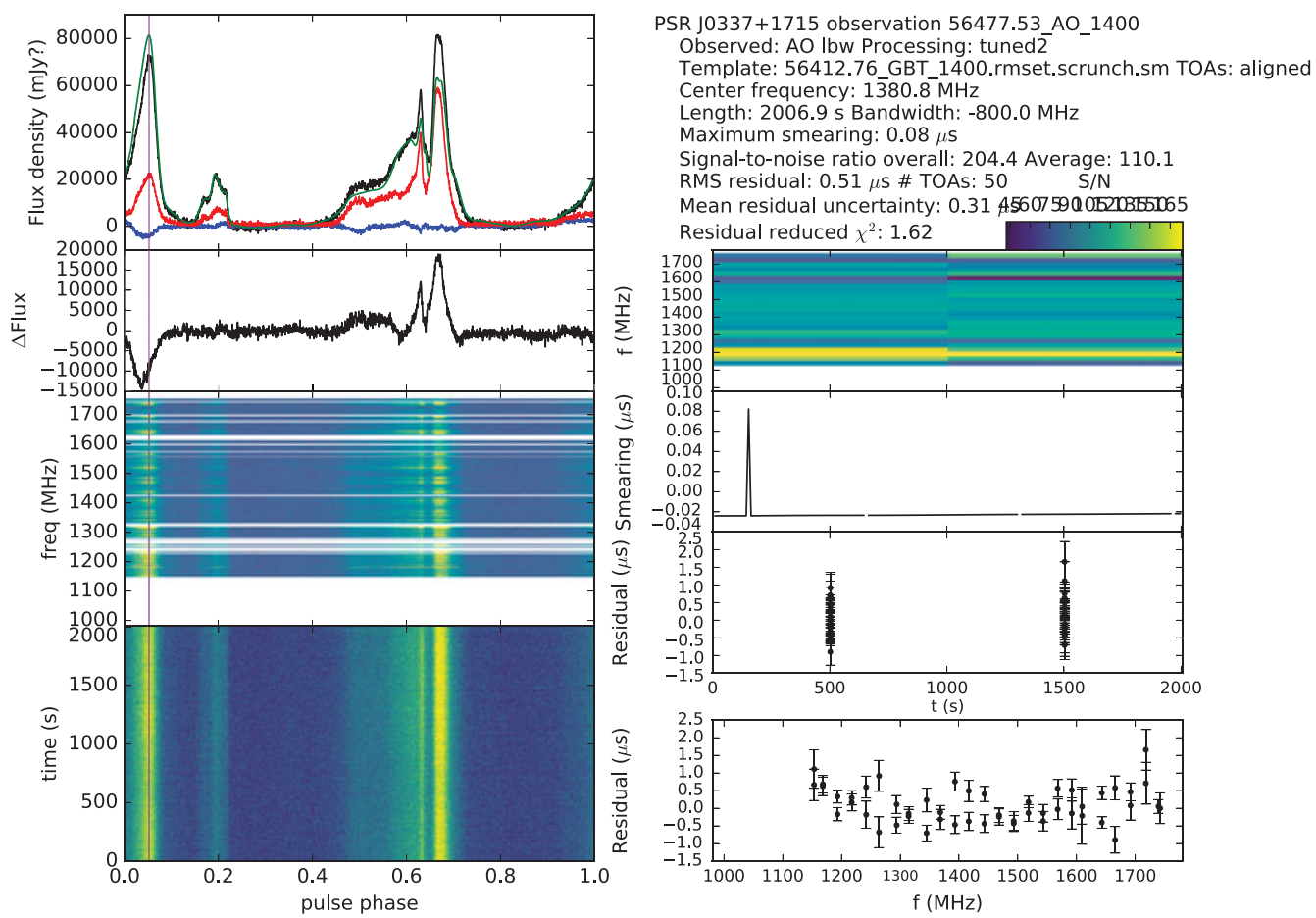

Figure 1. Summary plot for a processed Arecibo observation. Note the top left panel shows a substantial mismatch between the template (green) and the observed total-intensity profile (black); this is resolved by a suitable Mueller-matrix transformation. Left panels: average profile and template, difference between profile and template, profile as a function of frequency, profile as a function of time. Right panels: observation metadata, TOA signal-to-noise, ephemeris smearing, residual (with respect to a long-term ephemeris) as a function of time, residual as a function of frequency. Our processing pipeline generates such a plot for each observation; these are used for data quality monitoring and RFI excision.

\section{Fitting procedure}

Our primary fitting procedure is a Markov Chain Monte Carlo (MCMC) fit using the software package emcee (Foreman-Mackey et al. 2013); this code maintains a cloud of "walkers" that converge towards the posterior distribution and whose log-probabilities can be computed in parallel. We are therefore able to use up to a few hundred cores to carry out the fit; this is vital because each simulated orbit takes $30-60$ seconds. The MCMC fit yields an estimate of the posterior distribution, which we find to be essentially normal, as expected when all parameters are well-constrained. The exception to the constraints are the PPN parameters $\gamma$ and $\beta$, which we include for completeness but which are very poorly constrained by our data; to these we apply priors based on solarsystem tests.

In light of the normality of the posterior distribution, in principle Bayesian methods are unnecessary: a simple nonlinear least-squares fitting operation plus evaluation of the derivatives of the residuals with respect to all parameters would allow us to compute the same normal distribution. Unfortunately, the integrator operates near (but safely within) the limits of the 80-bit floating-point numbers we use, and so numerical roughness, combined with fairly strong parameter covariances, prevent ordinary nonlinear optimizers from reliably finding the minimum. Thus the MCMC method above serves as a more-resilient global optimizer, yielding inputs to the local optimizer that allow it to get 


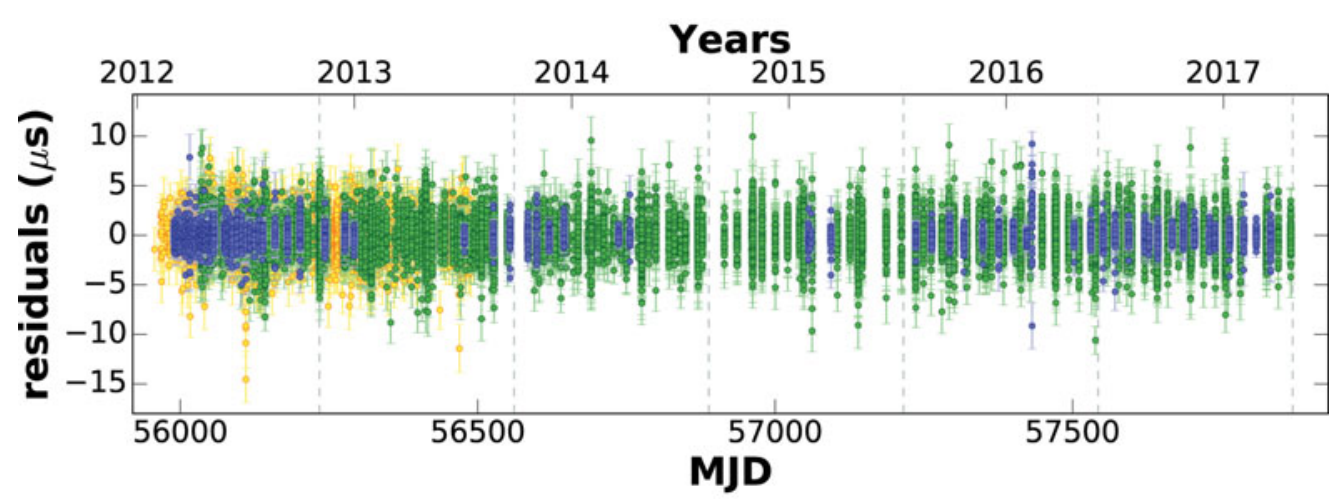

Figure 2. Residuals of the full numerical fit. Each color represents a different telescope: orange - Westerbork, green - GBT and blue - Arecibo. Dashed vertical lines represent ascending nodes of the outer orbit.

past obstacles and find the global minimum. When we calculate numerical derivatives using numdifftools, we find that the error region we infer from this frequentist method agrees with the error region we obtain from the Bayesian approach.

\section{Systematics}

Unfortunately, we find that some systematic effects have not been accounted for in our fitting procedure. The first hint of trouble is the reduced $\chi^{2}$ of the fit, which is approximately 1.3 for $\sim 27000$ degrees of freedom. We therefore examine our data, seeking structure in the residuals, particularly structure that might affect the fit value for our SEP violation parameter $\Delta$. Manual inspection of the residuals (see Figure 2) reveals no obvious trends, but with this many TOAs subtle effects too small to see in the residuals can nevertheless have substantial impacts on fit parameters. We therefore use a quasiFourier approach to measure structure that might affect $\Delta$. See Gusinskaia et al., this volume, for more detail.

\section{References}

Foreman-Mackey, D., Hogg, D. W., Lang, D., \& Goodman, J. 2013, PASP, 125, 306

Nordtvedt, K. 1985, ApJ, 297, 390

Ransom, S. M., Stairs, I. H., Archibald, A. M., et al. 2014, Nature, 505, 520

The NANOGrav Collaboration, Arzoumanian, Z., Brazier, A., et al. 2015, ApJ, 813, 65

van Straten, W. 2006, ApJ, 642, 1004 